\title{
Predictors of In-Hospital Mortality after Acute Ischemic Stroke in Subjects with and without Diabetes Mellitus
}

\author{
Athanasia Papazafiropoulou*, Alexios Sotiropoulos, Eystathios Skliros, Marina Kardara, \\ Anthi Kokolaki and Stavros Pappas
}

\author{
$3^{\text {rd }}$ Department of Internal Medicine and Center of Diabetes, General Hospital of Nikaia “Ag. Panteleimon” - Piraeus, \\ Greece
}

\begin{abstract}
Background: Diabetes mellitus is a well-established risk factor for ischemic stroke and is associated with increased in-hospital mortality. The aim of the present study was to determine the potential predictors of in-hospital mortality after an ischemic stroke in diabetic and nondiabetic subjects.

Methods: 159 diabetic subjects (66 males / 93 females, mean age \pm SD: $77.4 \pm 6.4$ years) and 159 non diabetic subjects ( 66 males / 93 females, mean age \pm SD: $77.3 \pm 5.2$ years) hospitalized for ischemic stroke were studied. Demographic characteristics and laboratory tests on admission as well as outcome were recorded. Brain computed tomography scan was performed in all study subjects.

Results: In-hospital death rates did not differ between the diabetic and the nondiabetic patients [36 (22.6\%) vs. 27 $(17.0 \%)$, respectively, $\mathrm{P}=0.20]$. In the diabetic study group multivariate analysis, after controlling for CRP, total cholesterol, LDL-C, urea and creatinine levels, demonstrated that death was related with WBC count (OR: 1.002, 95\% CI: 1.001-1.004, P = 0.005), glucose levels (OR: 1.007, 95\% CI: 1.002-1.012, P = 0.008), and UA levels (OR: $1.51,95 \% \mathrm{CI}$ : $1.003-2.260, \mathrm{P}=0.05)$. In the nondiabetic study group, after controlling for WBC count, $\mathrm{CRP}$, total cholesterol and LDL$\mathrm{C}$ levels, death was related only with glucose levels (OR: 1.016, 95\% CI: 1.001-1.031, P = 0.03).

Conclusions: WBC count and UA levels on hospital admission are independent predictors for in-hospital mortality in diabetic subjects with ischemic stroke. Plasma glucose levels are predictor for in-hospital mortality in both diabetic and nondiabetic subjects.
\end{abstract}

\section{INTRODUCTION}

It is well established that diabetic patients suffer often from macrovascular complications [1] while cardiovascular morbidity and mortality is particularly common in this group of patients [2]. A lot of studies have shown that diabetes mellitus (DM) is a major risk factor for increased mortality after acute ischemic stroke [3, 4] as well as after intracerebral hemorrhage [5]. Interestingly, a recent study showed that unknown diabetes is a strong risk factor for in-hospital mortality in subjects with acute ischemic stroke [6].

Studies in the general population have showed the predictive significance for increased mortality after acute stroke of different factors, including increased age [7], presence of DM [8], atrial fibrillation [9], increased hematocrit, leukocytosis [10], and hyperglycemia [11]. Studies, such as above, might help to determine factors influencing the outcome in subjects hospitalized for acute stroke. Therefore, the identification of predictors for in-hospital mortality might contribute to reduce death rates after acute stroke by enhancing the application of specific therapeutic strategies to the patients. However, the existing literature data, regarding the predic-

*Address correspondence to this author at the $3^{\text {rd }}$ Department of Internal Medicine and Center of Diabetes, General Hospital of Nikaia "Ag. Panteleimon" - Piraeus, 3 D.Mantouvalou Street, GR-184 54 Nikaia, Greece; Tel: +30 213207 6425; Fax: +30 213207 6426;

E-mail: pathan@ath.forthnet.gr tive significance of different factors for in-hospital mortality, especially in diabetic subjects, are limited.

Therefore, we conducted the present study in order to evaluate the prognostic value of different variables, including medical history and laboratory tests on admission, for inhospital mortality in diabetic and nondiabetic subjects with acute ischemic stroke.

\section{METHODS}

A total of 318 patients admitted to the Third Department of Internal Medicine (General Hospital of Nikaia, Athens, Greece) between June 2006 and December 2008 with acute ischemic stroke were included in the study. Only the patients with the onset of symptoms up to 12 hours before admission to the emergency room were recruited into the study. The ischemic stroke was confirmed in all patients by brain computed tomography (CT) scan. Stroke diagnosis was ascertained according to WHO criteria [12].

At baseline, demographic data (age, sex), history of diabetes mellitus, hypertension, hyperlipidemia, coronary artery disease (CAD), atrial fibrillation, and previous stroke, were obtained. The collection of the data was based on a history taken directly from the patients or their relatives and records from previous hospitalizations and diagnoses made during the present hospitalization. Patients were classified as having 
recognized diabetes if their medical records contained documentation of a previous history of diabetes, diagnosis of diabetes on admission, or the use of an oral antihyperglycemic agent or insulin at the time of hospital admission. Arterial hypertension as well as hyperlipidemia was recorded according to medical history and relevant drug treatment. CAD was defined as presence of angina, history of previous myocardial infarction, positive stress testing, revascularization procedures or stenosis $>50 \%$ at the coronary arteries. Atrial fibrillation was defined as the persistent replacement of consistent $\mathrm{P}$ waves by rapid oscillations or fibrillatory waves on the electrocardiogram. Previous stroke was defined as history of hospitalization for a syndrome of rapidly developing clinical signs of focal or global disturbance lasting for 24 hours or longer, attributed to ischemic or hemorrhagic lesions in the brain and verified with CT scan.

All patients underwent general and neurological examination. We recorded systolic (SBP) and diastolic blood pressures (DBP) on admission. Routine laboratory investigations were performed the day of admission to hospital, and included levels of glucose, lipid profile [total cholesterol, highdensity lipoprotein-cholesterol (HDL-C) and low-density lipoprotein-cholesterol (LDL-C), triglycerides], urea, creatinine, uric acid (UA) hematocrit, and white blood cell count (WBC). High sensitivity C-reactive protein (hsCRP) was determined using ADVIA 1650 (Bayer, Elkhart, IN, USA). All patients received treatment according to the current guidelines but none of them underwent thrombolysis or surgical treatment [13]. On discharge from hospital, the length of stay and the occurrence of death were recorded.

The study was approved by the local ethics committee. Informed consent form was given by patients themselves or by relatives.

\section{STATISTICAL ANALYSIS}

Statistical analysis was preformed using programs available in the SPSS statistical package (SPSS 15.0, Chicago, USA). All variables were tested for normal distribution of the data. Data are shown as mean $\pm \mathrm{SD}$, unless it is stated otherwise. A two sample $t$-test was used to assess differences in continuous variables, while a chi-square test was used for categorical variables. Univariate binary logistic analysis was performed to look for the relationship between death rates and the variables of interest in the sample population. Then, multivariate analysis was performed (backward stepwise method) to look for independent associations between death rates and the variables of interest. All independent variables in the multivariate analysis were tested for multicolinearity. $\mathrm{P}<0.05$ was considered statistically significant.

\section{RESULTS}

\section{Study Population Characteristics}

A total of 159 diabetic subjects (66 males / 93 females, mean age \pm SD: $77.4 \pm 6.4$ years) and equal number of non diabetic subjects (66 males / 93 females, mean age \pm SD: $77.3 \pm 5.2$ years) were enrolled in the study. The two study groups were matched by gender and age in order to avoid confounding factors to the statistical analysis. The demographic and clinical characteristics of the study subjects according to the presence of diabetes are showed on Table $\mathbf{1 .}$
The median (interquartile range) length of hospital stay was not different between the diabetic and the nondiabetic patients [6 (4-8) vs. 7 (4-8) days, respectively, $P=0.80$ ]. Inhospital death rates did not differ between the diabetic and the nondiabetic patients [36 (22.6\%) vs. $27(17.0 \%)$, respectively, $\mathrm{P}=0.20]$.

Participants with and without diabetes did not differ in terms of age, gender, presence of hypertension, atrial fibrillation, history of a previous stroke and CAD. As it was expected diabetic subjects had higher values of plasma glucose $(\mathrm{P}<0.001)$, and had more often hyperlipidemia $(\mathrm{P}=0.01)$ than their control counterparts. Also, diabetic subjects had higher values of plasma triglycerides levels $(\mathrm{P}=0.005)$, lower HDL-C levels $(\mathrm{P}=0.001)$ than nondiabetic subjects. No such difference was observed regarding total cholesterol and LDL-C levels between the study groups. Furthermore, diabetic subjects had higher values of plasma urea concentrations $(\mathrm{P}<0.001)$, plasma UA levels $(\mathrm{P}=0.002)$, WBC count $(\mathrm{P}=0.05)$, and lower hematocrit level $(\mathrm{P}=0.008)$ than nondiabetic subjects.

$18.4 \%$ of the diabetic subjects were on insulin treatment and $81.6 \%$ on antidiabetic tablets. In the diabetic study group the most prevalent cardiovascular risk factors were hypertension $(69.8 \%)$, CAD $(28.9 \%)$, and history of a previous stroke $(28.3 \%)$. In the nondiabetic study group the most prevalent cardiovascular risk factors were hypertension (66.7\%), atrial fibrillation $(27.0 \%)$, and history of a previous stroke $(23.9 \%)$.

\section{Subjects with Diabetes}

In the diabetic study group univariate logistic analysis showed that death was associated with hematocrit [odds ratio (OR): 0.91, 95\% Confidence Intervals (95\% CI): 0.85-0.97, $\mathrm{P}=0.005$ ], WBC count (OR: 1.002, 95\% CI: 1.001-1.004, $\mathrm{P}<0.001$ ), hsCRP (OR: $1.009,95 \% \mathrm{CI}: 1.003-1.015, \mathrm{P}=0$. 004), total cholesterol levels (OR: 0.98, 95\% CI: 0.97-0.99, $\mathrm{P}=0.03$ ), LDL-C levels (OR: 0.98, 95\% CI: 0.96-0.99, P = 0.007), glucose (OR: 1.004, 95\% CI: 1.001-1.006, $\mathrm{P}=0$. 006), urea levels (OR: 1.013, 95\% CI: 1.004-1.023, $\mathrm{P}=0$. 006), creatinine (OR: 2.79, 95\% CI: 1.46-5.32, $\mathrm{P}=0.002)$, and UA levels (OR: 1.42, 95\% CI: 1.14-1.79, P = 0.002). No any significant relationships were found between death and sex, age, SBP, DBP, history of hypertension, CAD, hyperlipidemia, previous stroke, atrial fibrillation, plasma HDL-C and tiglycerides levels (Table 2 ).

Multivariate analysis demonstrated, after controlling for hsCRP, total cholesterol, LDL-C, urea and creatinine levels, that death was related positively with WBC count (OR: 1.002, 95\% CI: 1.001-1.004, P = 0.005), glucose levels (OR: 1.007, 95\% CI: $1.002-1.012, \mathrm{P}=0.008)$, and UA levels (OR: $1.51,95 \%$ CI: $1.003-2.260, \mathrm{P}=0.05)$ (Table 2$)$.

\section{Subjects without Diabetes}

In the nondiabetic study group univariate logistic analysis showed that death was associated with WBC count (OR: 1.004, 95\% CI: 1.002-1.008, P = 0.005), hsCRP (OR: 1.008, 95\% CI: 1.003-1.014, $\mathrm{P}=0.003)$, total cholesterol levels (OR: 0.98, 95\% CI: 0.96-0.99, P = 0.04), LDL-C levels (OR: 0.97, 95\% CI: $0.96-0.99, \mathrm{P}=0.004)$, and glucose (OR: $1.018,95 \%$ CI: $1.005-1.030, \mathrm{P}=0.007)$. No any significant 
Table 1. Demographic and Clinical Characteristics of Subjects with and without Diabetes

\begin{tabular}{|c|c|c|c|}
\hline $\mathrm{n}(\%)$ & $159(50.0)$ & $159(50.0)$ & 1.00 \\
\hline Age (years) & $77.4 \pm 6.4$ & $77.3 \pm 5.2$ & 0.93 \\
\hline Systolic blood pressure $(\mathrm{mm} \mathrm{Hg})$ & $134.6 \pm 21.7$ & $136.7 \pm 24.1$ & 0.41 \\
\hline Glucose (mg / dl) & $207.7 \pm 138.9$ & $119.2 \pm 35.2$ & $<0.001$ \\
\hline Total cholesterol (mg / dl) & $178.3 \pm 45.6$ & $182.8 \pm 43.7$ & 0.42 \\
\hline HDL cholesterol (mg / dl) & $40.3 \pm 14.0$ & $46.6 \pm 15.9$ & 0.001 \\
\hline Creatinine (mg / dl) & $1.17 \pm 0.6$ & $1.06 \pm 0.71$ & 0.16 \\
\hline Uric acid (mg / dl) & $5.8 \pm 2.2$ & $5.1 \pm 1.7$ & 0.002 \\
\hline $\operatorname{WBC}\left(10^{9} / 1\right)$ & $9,860.6 \pm 4,418.1$ & $8,927.5 \pm 4,082.7$ & 0.05 \\
\hline $\mathrm{CRP}(\mathrm{mg} / \mathrm{dl})$ & $34.0 \pm 18.7$ & $35.5 \pm 16.2$ & 0.85 \\
\hline Hematocrit (\%) & $38.0 \pm 5.8$ & $39.6 \pm 5.0$ & 0.008 \\
\hline Hypertension (yes) n (\%) & $111(69.8)$ & $106(66.7)$ & 0.55 \\
\hline CAD (yes) n (\%) & $46(28.9)$ & $36(22.6)$ & 0.20 \\
\hline Insulin & $27(18.4)$ & - & - \\
\hline
\end{tabular}

*Median values (interquartile range). P values for the comparison between subjects with and without diabetes by independent samples t-test for continuous variables or by Pearson $\chi^{2}$ for nominal variables.

HDL: high density lipoprotein; LDL: low density lipoprotein; WBC: white blood cell count; CRP: C reactive protein; CAD: coronary artery disease

relationships were found between death and sex, age, SBP, DBP, history of hypertension, CAD, hyperlipidemia, previous stroke, atrial fibrillation, urea levels, creatinine, UA levels, plasma HDL-C and tiglycerides levels (Table 3).

Multivariate analysis demonstrated, after controlling for WBC count, hsCRP, total cholesterol and LDL-C levels, that death was related positively only with glucose levels (OR: 1.016, 95\% CI: 1.001-1.031, P = 0.03) (Table 3).

\section{DISCUSSION}

The main finding of the present study was that WBC count, plasma glucose and UA levels on admission were independent predictors for in-hospital mortality in diabetic subjects. In the nondiabetic group plasma glucose levels was the only independent predictor for in-hospital mortality.
In diabetic subjects elevated serum UA levels are related with increased risk of future stroke $[14,15]$. A recent study showed that in type 2 diabetic subjects elevated plasma UA levels were associated with increased arterial stiffness, which is a well-recognised cardiovascular risk factor [16]. Several studies in the general population showed an effect of serum UA levels on prediction of stroke outcomes [17-19]. Two studies have showed that elevated levels of UA were independently associated with an increased risk of early death after acute stroke $[17,18]$. In addition, in patients admitted with stroke, serum UA was an independent predictor of future cardiovascular events in the next 2 years [19]. Furthermore, a study showed that elevated UA was associated with an increased risk for acute stroke in elderly individuals [20]. A study in healthy postmenopausal women showed that serum UA levels, independently of other cardiovascular risk 
Table 2. Univariate and Multivariate Logistic Analysis: The Association between Various Parameters with Death in Subjects with Diabetes

\begin{tabular}{|c|c|c|c|c|c|c|}
\hline & \multicolumn{3}{|c|}{ Univariate Analysis } & \multicolumn{3}{|c|}{ Multivariate Analysis } \\
\hline & Odds Ratio & 95\% Confidence Intervals & P-Value & Odds Ratio & 95\% Confidence Intervals & P-Value \\
\hline Hematocrit & 0.91 & $0.85-0.97$ & 0.005 & - & - & - \\
\hline WBC count & 1.002 & $1.001-1.004$ & $<0.001$ & 1.002 & $1.001-1.004$ & 0.005 \\
\hline Total cholesterol & 0.98 & $0.97-0.99$ & 0.03 & - & - & - \\
\hline LDL- cholesterol & 0.98 & $0.96-0.99$ & 0.007 & - & - & - \\
\hline Glucose & 1.004 & $1.001-1.006$ & 0.006 & 1.007 & $1.002-1.012$ & 0.008 \\
\hline Urea levels & 1.013 & $1.004-1.023$ & 0.006 & - & - & - \\
\hline
\end{tabular}

Table 3. Univariate and Multivariate Logistic Analysis: The Association between Various Parameters with Death in Subjects without Diabetes

\begin{tabular}{|c|c|c|c|c|c|c|}
\hline & \multicolumn{3}{|c|}{ Univariate Analysis } & \multicolumn{2}{c|}{ Multivariate Analysis } \\
\cline { 2 - 6 } & Odds Ratio & 95\% Confidence Intervals & P-Value & Odds Ratio & 95\% Confidence Intervals & P-Value \\
\hline \hline WBC count & 1.004 & $1.002-1.008$ & 0.005 & - & - & - \\
\hline CRP & 1.008 & $1.003-1.014$ & 0.003 & - & - \\
\hline Total cholesterol & 0.98 & $0.96-0.99$ & 0.04 & - & - \\
\hline LDL- cholesterol & 0.97 & $0.96-0.99$ & 0.004 & - & - \\
\hline Glucose & 1.018 & $1.005-1.030$ & 0.007 & 1.016 & - \\
\hline
\end{tabular}

factors, were associated with intima-media thickness (IMT), a well known marker of cardiovascular disease [21].

However, a study showed that in patients with ischemic stroke there was a $12 \%$ increase in the odds of good clinical outcome for each milligram per deciliter increase of UA [22]. The explanation given by the authors was that the antioxidant capacity of UA is an independent factor that ameliorates the clinical prognosis of patients with acute ischemic stroke [22].

A lot of studies have shown that WBC count measured at the time of hospital admission was associated with greater initial stroke severity $[10,23,24]$ and might have a prognostic value in acute stroke survivors [10, 25]. Subjects with increased WBC count have excess risk of ischemic strokes independently of other cardiovascular risk factors [26]. Furthermore, WBC count has been shown to contribute to the initiation and further development of ischemic stroke [27, 28]. Two studies showed that WBC count measured in patients during the first 24-hours after the stroke onset was significantly elevated in comparison to healthy controls [24,
29]. However, the observed increased WBC count did not have any prognostic value for the outcome of the patients [24, 29].

It is well established that blood glucose increases after the onset of acute stroke and the increase is related to the severity of the stroke [30]. A systematically review of the existing data showed that in patients with no history of diabetes who have an ischemic stroke, even moderately elevated glucose levels are associated with both a 3-fold higher risk of short-term mortality and an increased risk of poor functional recovery compared with lower glucose levels [31]. Many studies have shown higher mean admission glucose level in nonsurvivors of stroke compared with survivors [32-34]. Furthermore, two large studies demonstrated that glucose level at hospital admission was a significant predictor of mortality [35] or poor functional recovery [36] after stroke independent of other prognostic factors. In addition, a study showed that persistent hyperglycemia was an independent determinant of infarct expansion and was associated with worse functional outcome [37]. 


\section{CONCLUSION}

In conclusion, WBC count, and UA levels on hospital admission are independent predictors for in-hospital mortality in diabetic subjects with ischemic stroke. Plasma glucose levels are predictor for in-hospital mortality in both diabetic and nondiabetic subjects. Therefore, early interventions aiming the above factors might have a positive result to the outcome of patients with ischemic stroke.

\section{ABBREVIATIONS}

$\begin{array}{ll}\mathrm{CAD} & =\text { Coronary artery disease } \\ \mathrm{CI} & =\text { Confidence Intervals } \\ \mathrm{CT} & =\text { Diastolic blood pressure } \\ \mathrm{DBP} & =\text { Diabetes mellitus } \\ \mathrm{DM} & \text { High sensitivity C-reactive protein } \\ \text { hSCRP } & \text { High-density lipoprotein-cholesterol } \\ \text { HDL-C } & =\text { Intima-media thickness } \\ \text { IMT } & =\text { Low-density lipoprotein-cholesterol } \\ \text { LDL-C } & \text { Odds ratio } \\ \text { OR } & =\text { Systolic blood pressure } \\ \text { SBP } & =\text { Uric acid } \\ \text { UA } & =\text { White blood cell count }\end{array}$

\section{REFERENCES}

[1] Stamler J, Vaccaro O, Neaton JD, Wentworth D. Diabetes, other risk factors, and 12-yr cardiovascular mortality for men screened in the Multiple Risk Factor Intervention Trial. Diabetes Care 1993; 16: 434-44.

[2] Kleinman JC, Donahue RP, Harris MI, Finucane FF, Madans JH, Brock DB. Mortality among diabetics in a national sample. Am J Epidemiol 1988; 128: 389-401.

[3] Weir CJ, Murray GD, Dyker AG, Lees KR. Is hyperglycaemia an independent predictor of poor outcome after acute stroke? Results of a long-term follow-up study. BMJ 1997; 314: 1303-6.

[4] Arboix A, Massons J, García-Eroles L, Oliveres M, Targa C. Diabetes is an independent risk factor for in-hospital mortality from acute spontaneous intracerebral hemorrhage. Diabetes Care 2000; 23: 1527-32.

[5] Di Bonito P, Di Fraia L, Di Gennaro L, et al. Impact of known and unknown diabetes on in-hospital mortality from ischemic stroke. Nutr Metab Cardiovasc Dis 2003; 13: 148-53

[6] Zuliani G, Cherubini A, Ranzini M, Ruggiero C, Atti AR, Fellin R. Risk factors for short-term mortality in older subjects with acute ischemic stroke. Gerontology 2006; 52: 231-6.

[7] Benedetti MD, Benedetti M, Stenta G, Costa B, Fiaschi A. Shortterm prognosis of stroke in a clinical series of 94 patients. Ital J Neurol Sci 1993; 14: 121-7.

[8] Kushner M, Nencini P, Reivich M, et al. Relation of hyperglycemia early in ischemic brain infarction to cerebral anatomy, metabolism and clinical outcome. Ann Neurol 1990; 28: 129-35.

[9] Candelise L, Pinardi G, Morabito A. Mortality in acute stroke with atrial fibrillation. The Italian Acute Stroke Study Group. Stroke 1991; 22: 169-74.

[10] Czlonkowska A, Ryglewicz D, Lechowicz W. Basic analytical parameters as predictive factors for 30-day case fatality rate in stroke. Acta Neurol Scand 1997; 95: 121-4.

[11] Candelise L, Landi G, Orazio EN, Boccardi E. Prognostic significance of hyperglycemia in acute stroke. Arch Neurol 1985; 24: $661-3$
[12] Recommendations on stroke prevention, diagnosis, and therapy. Report of the WHO Task Force on stroke and other cerebrovascular disorders. Stroke 1989; 20: 1407-31.

[13] Adams H, Adams R, Del Zoppo G, Goldstein LB. Guidelines for the early management of patients with ischemic stroke: 2005 Guidelines update: A Scientific Statement from the Stroke Council of the American Heart Association/American Stroke Association. Stroke 2005; 36: 916-23

[14] Lehto S, Niskanen L, Ronnemaa T, Laakso M. Serum uric acid is a strong predictor of stroke in patients with non-insulin-dependent diabetes mellitus. Stroke 1998; 29: 635- 9.

[15] Seghieri G, Moruzzo D, Fascetti S, et al. Increase in serum uric acid is selectively associated with stroke in type 2 diabetes. Diabe tes Care 2002; 25: 1095.

[16] Papazafiropoulou A, Tentolouris N, Moyssakis I, Perrea D, Katsilambros N. The potential effect of some newer risk factors for atherosclerosis on aortic distensibility in subjects with and without type 2 diabetes. Diabetes Care 2006; 29: 1926-8.

[17] Karagiannis A, Mikhailidis DP, Tziomalos K, et al. Serum uric acid as an independent predictor of early death after acute stroke. Circ J 2007; 71: 1120-7.

[18] Cherubini A, Polidori MC, Bregnocchi M, et al. Antioxidant profile and early outcome in stroke patients. Stroke 2000; 31: 2295300 .

[19] Weir CJ, Muir SW, Walters MR, Lees KR. Serum urate as an independent predictor of poor outcome and future vascular events after acute stroke. Stroke 2003; 34: 1951-7.

[20] Milionis HJ, Kalantzi KJ, Goudevenos JA, Seferiadis K, Mikhailidis DP, Elisaf MS. Serum uric acid levels and risk for acute ischaemic non-embolic stroke in elderly subjects. J Intern Med 2005; 258: 435-41.

[21] Montalcini T, Gorgone G, Gazzaruso C, Sesti G, Perticone F, Pujia A. Relation between serum uric acid and carotid intima-media thickness in healthy postmenopausal women. Intern Emerg Med 2007; 2: 19-23.

[22] Chamorro A, Obach V, Cervera A, Revilla M, Deulofeu R, Aponte JH. Prognostic significance of uric acid serum concentration in patients with acute ischemic stroke. Stroke 2002; 33: 1048-52.

[23] Kazmierski R, Guzik P, Ambrosius W, Ciesielska A, Moskal J, Kozubski W. Predictive value of white blood cell count on admis sion for in-hospital mortality in acute stroke patients. Clin Neurol Neurosurg 2004; 107: 38-43

[24] Woo J, Lau E, Kay R, et al. A case control study of some hematological and biochemical variables in acute stroke and their prognostic value. Neuroepidemiology 1990; 9: 315-20.

[25] Hénon H, Godefroy O, Leys D, et al. Early predictors of death and disability after acute cerebral ischemic stroke event. Stroke 1995 26: 392-8.

[26] Prentice RL, Szatrowski TP, Kato H, Mason MW. Leukocyte counts and cerebrovascular disease. J Chronic Dis 1982; 35: 703 14.

[27] Akopov SE, Simonian NA, Grigorian GS. Dynamics of polymorphonuclear leukocytes accumulation in acute cerebral infarction and their correlation with brain tissue damage. Stroke 1996; 27: 1739-43

[28] Bednar MM, Raymond S, McAuliffe T, Lodge PA, Gross GG. The role of neutrophils and platelets in a rabbit model of thromboembolic stroke. Stroke 1991; 22: 2120-6.

[29] Silvestrini M, Pietroiusti A, Troisi E, et al. Leukocyte count and aggregation during the evolution of cerebral ischemic injury. Cerebrovasc Dis 1998; 8: 305-9.

[30] Christensen H, Boysen G. Blood glucose increases early after stroke onset: a study on serial measurements of blood glucose in acute stroke. Eur J Neurol 2002; 9: 297-301.

[31] Capes SE, Hunt D, Malmberg K, Pathak P, Gerstein HC. Stress hyperglycemia and prognosis of stroke in nondiabetic and diabetic patients: a systematic overview. Stroke 2001; 32: 2426-32.

[32] Tracey F, Crawford VLS, Lawson JT, Buchanan KD, Stout RW. Hyperglycaemia and mortality from acute stroke. Q J Med 1993; 86: 439-46.

[33] M'Buyamba-Kabangu J-R, Longo-Mbenza B, Tambwe MJ, Dikassa LN, Mbala-Mukendi M. J-shaped relationship between mortality and admission blood pressure in black patients with acute stroke. J Hypertens 1995; 13: 1863-8. 
[34] Murros K, Fogelholm R, Kettunen S, Vuorela A-L, Valve J. Blood glucose, glycosylated haemoglobin, and outcome of ischemic brain infarction. J Neurol Sci 1992; 111: 59-64.

[35] Moulin T, Tatu L, Crepin-Leblond T, Chavot D, Berges S, Rumbach L. The Besançon Stroke Registry: an acute stroke registry of 2500 consecutive patients. Eur Neurol 1997; 38: 10 -20.
[36] Bruno A, Biller J, Adams HP, et al. Acute blood glucose level and outcome from ischemic stroke. Trial of ORG 10172 in acute stroke treatment (TOAST) investigators. Neurology 1999; 52: 280-4.

[37] Baird TA, Parsons MW, Phanh T, et al. Persistent poststroke hyperglycemia is independently associated with infarct expansion and worse clinical outcome. Stroke 2003; 34: 2208-14.

(C) Papazafiropoulou et al.; Licensee Bentham Open.

This is an open access article licensed under the terms of the Creative Commons Attribution Non-Commercial License (http: //creativecommons.org/licenses/ by-nc/3.0/) which permits unrestricted, non-commercial use, distribution and reproduction in any medium, provided the work is properly cited. 